Keywords: Delusional Disorder; Paranoia; Affective symptoms; Depression; Antidepressants.

\title{
Delusional disorder: An overview of affective symptoms and antidepressant use
}

\section{Alexandre González-Rodríguez* \\ Oriol Molina-Andreu** \\ Víctor Navarro Odriozola*** \\ Cristóbal Gastó Ferrer*** \\ Rafael Penadés**** \\ Rosa Catalán****}

* Department of Psychiatry and Clinical Psychology, Hospital Clinic of Barcelona, University of Barcelona,

Barcelona

** Department of Psychiatry, Hospital Universitari Mútua de Terrassa, Terrassa

*** Department of Psychiatry and Clinical Psychology, Hospital Clinic of Barcelona IDIBAPS, CIBERSAM, University of Barcelona, Barcelona

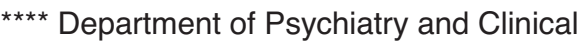
Psychology, Hospital Clinic of Barcelona, CIBERSAM, University of Barcelona, Barcelona

SPAIN

\begin{abstract}
Background and Objectives: In clinical practice, the coexistence of delusions and affective symptoms is often observed. The current Diagnostic and Statistical Manual of Mental Disorders, fourth edition- Text Revision (DSM-IV-TR) accepts the existence of mood disturbances in delusional disorder. Little research has been done regarding this field and prevalence rates are unclear. Our purpose was to review the frequency of affective symptoms in delusional disorder patients and to identify the proportion of these patients that are reported to be treated with antidepressants.

Methods: Three electronic databases were searched from 1980 to July 2012: MEDLINE, PsycINFO and PubMed, using the following keywords: delusional disorder, depression, affective illness, antidepressants and paranoia.

Results: A high frequency of affective disturbances was found in this population (21$55.8 \%$ ). In contrast, a low antidepressants prescription rate was shown. When focusing on delusional disorder somatic subtype, a particularly high mood disorder comorbidity rate was described and a good efficacy of antidepressant drugs was reported, in monotherapy or in combination with antipsychotics.
\end{abstract}


Conclusions: A high frequency of comorbid depression in delusional disorder and a low antidepressants prescription rate could be concluded. Further studies are needed to better clarify the role of mood symptoms in clinical practice among delusional disorder patients.

Received: 15 March 2013

Revised: 3 September 2013

Accepted: 27 September 2013

\section{Background and objectives}

Kraepelin emphasized the clinical separation of paranoia from dementia precox and manic-depressive insanity ${ }^{1}$. However, several psychiatrists continue proposing that delusional disorder (DD) is a subtype of schizophrenia. On the other hand, some researchers, such as Specht, suggest that DD is only a form of affective illness ${ }^{2,3}$. DD (paranoia) was introduced by Winokur and first implemented in the DSM-III-R ${ }^{4}$. As established in the Diagnostic and Statistical Manual of Mental Disorders- Fourth Edition, Text Revision (DSM-IV-TR $)^{5}$ delusional ideas in DD must be present for at least one month and criterion A for schizophrenia cannot be present. Affective symptoms can occur simultaneously, but they must be brief and related to the delusional theme.

Etiological factors of DD have not been yet elucidated $^{6}$. However, the influence of genetic, biological (hyperdopaminergic states) and psychological factors have been widely considered in the scientific literature. In particular, blockade of the 5-HT receptors and specifically of 5-TH2A has been hypothesized to be relevant to understand physiopathology in delusional parasitosis 7,8 .

Rates of psychiatric comorbidity in patients with DD have been found to be high, being affective disorders the most common comorbid entity ${ }^{9,10}$. These patients show an early onset of illness, greater severity of general psychopathology and better insight than subjects without comorbidity ${ }^{9,11}$.

The pharmacotherapy of DD has been poorly studied. The treatment of DD would involve both pharmacotherapy ${ }^{12}$ and psychotherapy, when necessary ${ }^{13}$. Treatment alternatives may include antidepressants, antipsychotics or a combination of both. Despite this, several studies indicate a low rate of antidepressant prescription.

Our goal was to summarize the most important findings regarding the prevalence of depressive comorbidity in DD and the frequency of antidepressant use in this population, to provide suggestions for a better clinical management.

\section{Methods}

Three electronic databases: MEDLINE, PsycINFO and PubMed were searched from 1980 to July 2012 in order to examine the prevalence of depressive comorbidity in DD, frequency and reasons for antidepressant prescription. The following keywords were used: delusional disorder, depression, affective illness, antidepressants and paranoia. These searches were not restricted to publication type or design of the study and all relevant papers in English, German and Spanish were 
included. In addition, relevant abstracts related to the search terms were obtained to identify full-text articles.

Studies and case reports were only included if they met the following criteria: (1) be an original publication in a peer-review journal, (2) studied demographic and clinical variables in DD, (3) included information regarding depressive comorbidity and antidepressant use in DD.

Depressive comorbidity was defined as the diagnosis of a depressive disorder, as concurrent with the presence of DD.

\section{Results}

We report our findings by using the keywords previously mentioned, and the total number of studies which have been identified.

"Delusional disorder and depression/depressive illness": 1485 articles can be found regarding comorbid depression and DD. "Delusional disorder and antidepressants": 382 papers were found on DD and antidepressants use.

Two cases of organic DD were identified and excluded due to several reasons. Both patients were diagnosed as having a DD somatic type, also known as monosymptomatic hypochondriacal psychosis, which was related to medical conditions such as a stroke with delusions of parasitosis and jealousy. In one case, antidepressant therapy was not completely described, in the second case, quetiapine was combinated with sertraline in a patient with a history of stroke.

With our initial selection strategy, a total of 34 articles were identified: 14 case reports, 10 case-register, retrospective or prospective studies and 10 reviews or systematic reviews.
Demographic data, clinical features, DD subtype, presence or absence of comorbid depressive disorder and prescription rates of antidepressants can be found in Tables 1 to 2 .

Manschreck and co-workers ${ }^{14}$ reviewed all published reports on DD from 1994 to 2004, including 224 case descriptions. When attention was focused on comorbid conditions in $\mathrm{DD}$, the authors reported a prevalence of depression in 51 cases $(22.76 \%)$. In a previous systematic review, Kendler ${ }^{1}$ summarized demographic characteristics of paranoid psychoses and compared them with those found for schizophrenia and affective illness. Seventeen reports were reviewed from 1912 to 1978 and demographic data on DD available in hospital admissions were included. Like affective illness, DD was predominantly an illness of middle to late adult life, usually occurring in married status patients. However, in the systematic review mentioned above, the prevalence of depressive symptoms in this population was not described.

In a 4-year prevalence study, Yassa and Suranyi-Cadotte ${ }^{15}$ reported 13 cases of DD patients, where $23 \%$ of them showed depressive symptoms at the index episode (first admission) and no patients received antidepressant therapy.

An Italian Study conducted by Marino and colleagues $^{11}$ found a high frequency of affective comorbidity $(50.7 \%)$ and identified a clinical group of DD patients that had a recurrent form of mood disturbance $(35.2 \%)$.

A retrospective study of 86 DD outpatients was conducted by Hsiao and co-workers ${ }^{16}$. Thirty-seven cases $(43 \%)$ had a concurrent depression at their first psychiatric appointment. No statistically significant differences in terms of sociodemographic and clinical features were found between patients with depressive symptoms or without depressive symptoms. 


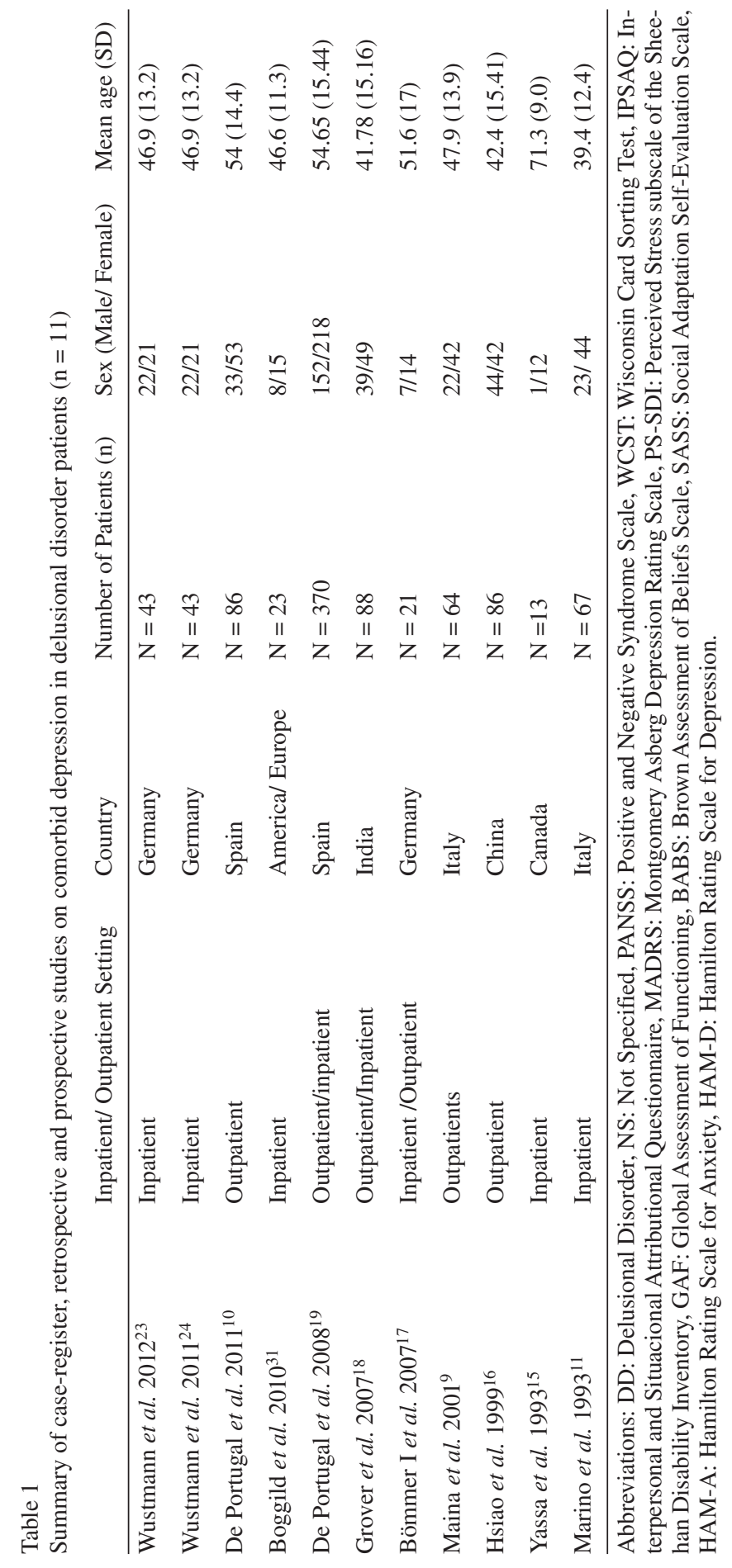




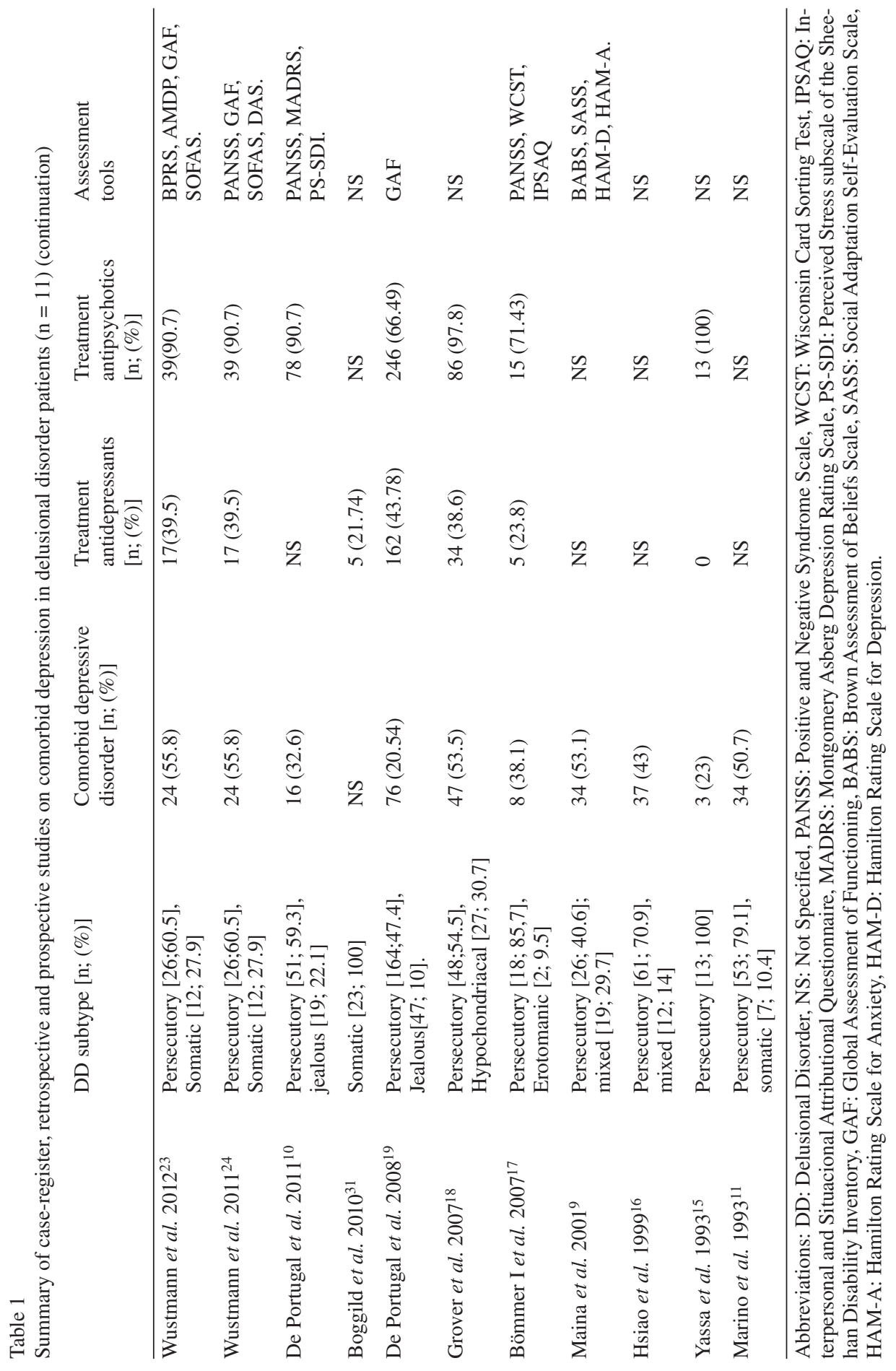


ALEXANDRE GONZÁLEZ-RODRÍGUEZ ET AL.

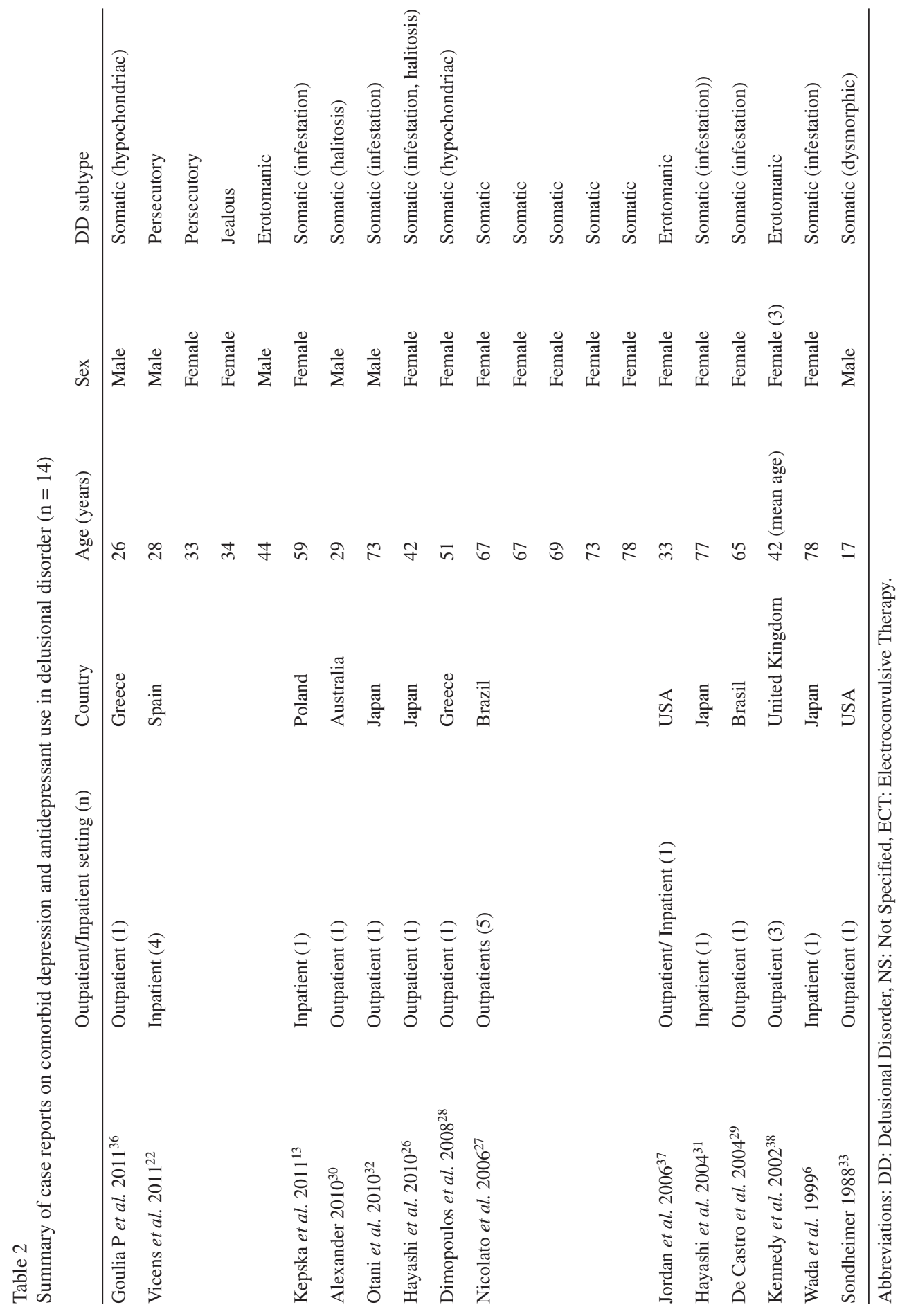


DELUSIONAL DISORDER AND DEPRESSION 271

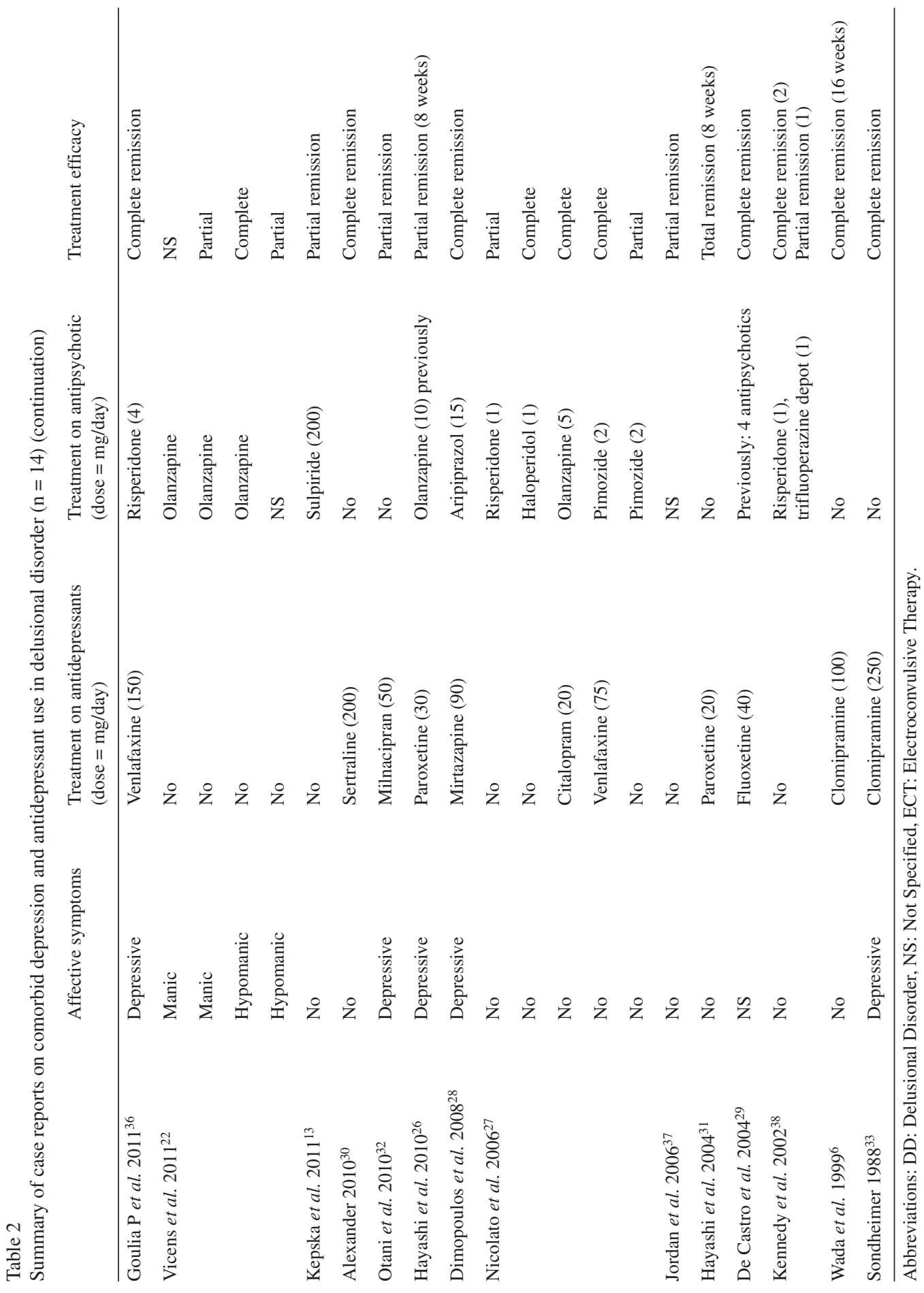


In a prospective observational study, Maina and colleagues ${ }^{9}$ investigated other comorbid axis I disorders in a sample of 64 DD patients. Seventy-two per cent of the sample had at least a comorbid psychiatric diagnosis and fiftythree per cent presented at least one comorbid mood disorder, being the most frequently encountered major depression (35.9\%). DD patients with persecutory type presented in most cases a coexisting disorder. Overall, the authors observed that patients with at least one comorbid disorder had an earlier age at onset of disorder and attended a first psychiatric consultation at an earlier age.

Bömmer I and Brüne $\mathrm{M}^{17}$ examined 21 patients with a diagnosis of DD according to ICD-10, and compared them with 22 healthy controls. PANSS for psychopathology, Wisconsin Card Sorting Test for neuropsychological aspects and Internal, Interpersonal and Situational Attribution Questionnaire were assessed in both groups. Patients with DD made more perseverative errors in the Wisconsin Card Sorting Test compared with healthy controls, but these differences were significant only in patients with a comorbid depression. The authors of this study concluded that these patients tended to show a depression attributional style. Moreover, it was suggested that a comorbid depressive disorder in DD patients is warranted and antidepressant treatment could be a useful option in these patients.

From India, Grover and co-workers ${ }^{18}$ carried out a retrospective study on 88 patients with DD, observing that $53.5 \%$ of the sample had a comorbid depressive disorder. Two patients were treated with antidepressants in monotherapy and 32 subjects received a combination of antipsychotics and antidepressant therapy. This study suggested that patients with comorbidity had an earlier age at onset, were at a younger age at their first psychiatric consultation and showed a higher compli- ance at follow-up appointments. Patients with DD jealous type had a higher prevalence of comorbidity, but the presence of depressive symptoms was not specified by the authors.

In a case-register study based on all patients that attended a community mental health service during a three-year period, de Portugal et al. ${ }^{19}$ found that $20.54 \%$ of DD patients presented a comorbid depressive disorder and forty-four per cent of the sample were treated with antidepressants at follow-up appointments. Persecutory and jealous types were significantly associated with higher antidepressant use and personal history of depression in comparison to other types. The same authors assessed depressive symptoms by using the Montgomery-Asberg Depression Rating Scale (MADRS) in DD patients, which were included in the DELIREMP study ${ }^{20}$. DD patients with grandiose type symptoms showed significantly lower depressive symptoms scores than patients with other types. Gender differences in presence and severity of depression were not found in another study ${ }^{21}$. At the same time, Vicens and co-workers ${ }^{22}$ published 2 cases of manic episodes and 2 cases of hypomanic symptoms in a clinical group of 4 patients with DD.

When focusing on the clinical correlates and comorbidity in DD, de Portugal et al. ${ }^{10}$ described a sample of 86 DD outpatients, where $46 \%$ of the sample had at least one psychiatric comorbid condition. Depressive disorders were found to be the most common comorbid entity and "pure" DD patients showed an impaired general functioning than DD subjects without psychiatric comorbidity.

Wustmann and co-workers ${ }^{23}$ identified all consecutive DD cases that were treated as inpatients at the Department of Psychiatry, Psychotherapy and Psychosomatics in HalleWittenberg from 1994 to 2008. Thirty-nine per cent of the patients received antidepressants and more than a half of the patients had 
depressive symptoms. Findings of a longitudinal 43-case study, carried out by the same authors, did not show differences between genders according to the type of DD and the frequency of depressive symptoms ${ }^{24}$.

When focusing on DD types and comorbidity, several case reports can be found. Somatic and erotomanic are the most frequently encountered types and antidepressant use and response rates were accurately described in these studies. Selective serotonin reuptake inhibitors (SSRIs) are the antidepressants most commonly prescribed in DD somatic type.

Hayashi et al. ${ }^{25}$ presented a 77-year-old woman with delusional infestation associated with a comorbid depressive syndrome, who was admitted to a psychiatric ward and treated with paroxetine $20 \mathrm{mg} /$ day. Total remission of symptoms was shown after 8 weeks of treatment. Paroxetine was suggested to be a successful treatment for DD, somatic type. In $2010^{26}$, the same authors presented the case of a 42-year-old female with DD somatic type and delusions of halitosis and body odor. She had a severe depression in relationship to somatic delusions, and a partial response was described after an 8-week treatment with paroxetine $30 \mathrm{mg}$ /day. In the last decade, Nicolato R et al. ${ }^{27}$ identified 4 cases of patients with DD somatic type, all of them treated with antipsychotics. Two patients received a combination of an antipsychotic and an antidepressant, and complete remission of the psychotic symptoms was observed. No mood disturbance was described by the authors in these patients. Simultaneously, Dimopoulos et al. ${ }^{28}$ presented a case of a female with DD somatic type, who responded to a combination of aripiprazol and mirtazapine.

In regards to fluoxetine ${ }^{29}$, a case of a female who presented a DD was described, having no clinical improvement when treated consecutively with pimozide, trifluoperazine, haloperi- dol and risperidone. After two months on fluoxetine, the patient remained asymptomatic. Alexander ${ }^{30}$ reported a case of a 29 -year-old software engineer with a 2-year history of delusional halitosis that did not improve with olanzapine and risperidone. Because of this lack of response, sertraline was prescribed in combination with cognitive and behavior therapy observing a total remission after 8 weeks. In another study, twenty-three cases of DD somatic type were identified by Boggild et $a l .{ }^{31}$ and were included in a prospective follow-up study. In 5 of them, antidepressants were prescribed in combination with an atypical antipsychotic. Depressive comorbidity was not described by the authors.

In another case report, Otani and colleagues ${ }^{32}$ presented a case of a 73-year-old male with the same delusional thought and concurrent depressive mood. The patient was treated with milnacipran $50 \mathrm{mg} /$ day and a significant clinical improvement was achieved.

Clomipramine was shown to improve a DD somatic-type in a 17-year-old outpatient $^{33}$, who was reported to present dysthymic mood, and in a 78-year-old female patient admitted to a psychiatric ward ${ }^{6}$.

Within the erotomanic type, Rudden et al. ${ }^{34}$ performed a comparison of 28 erotomanic patients with a group of 80 non-erotomanic subjects, 16 of them being diagnosed as DD. 19 patients of the total sample received antidepressant treatment. However, no antidepressant prescription rates or clinical comorbidity were described by diagnostic group.

\section{Discussion}

We have carried out an overview of published evidence regarding the frequency of affective symptoms and antidepressant use in DD patients. 
As it has been reported in schizophrenia, depressive symptoms are common in patients with DD and the presence of these symptoms could imply a worse prognosis in these patients and an increased suicide risk $^{39}$. As Maina et al. suggested ${ }^{9}$, this review could strengthen the notion that DD is associated with affective disorders in a high number of patients. However, there are conflicting data concerning the prevalence of depressive symptoms. Some authors have reported a prevalence of depressive symptoms in DD patients around $21 \%{ }^{19}$. On the other hand, Wustmann et al. ${ }^{23}$ found that DD patients had depressive comorbidity in $55.8 \%$ of all cases.

Evaluation of depressive symptoms was found to be lower among grandiose DD patients in comparison with persecutory or jealous types. No statistically significant gender differences could be found in the presence and severity of depression ${ }^{21}$. Nonetheless, differences in frequency of affective symptoms by delusional type could not be analyzed due to the heterogeneity of the methodology used in the reviewed studies.

Few studies have focused on differences of age at onset in DD patients with and without comorbidity ${ }^{35}$. Patients with comorbid depression and DD have an earlier age of onset, a more severe course of illness and yet greater insight than those without such condition ${ }^{9}$. These findings are important as an earlier diagnosis in patients with DD and depressive comorbidity may allow an adequate treatment of the disorder and better prevention of suicidal ideation and suicidal behaviour which have been reported to appear in chronic psychosis with depressive symptoms, such as in schizophrenia.

As it has been reported in the reviewed literature, a minority of subjects with DD received antidepressants, even when depression was associated as concurrent. Furthermore, no data can be found to support the recom- mendations about the use of antidepressants in DD as the main treatment or as an alternative. Grover et al. $^{18}$ suggested that this low antidepressant use probably could be explained by the tendency of some clinicians to not treat depression, when it is related to a DD diagnosis.

In spite of lack of data on treatment efficacy, recent findings suggest that antidepressants may be useful in the treatment of psychosis with or without comorbid depression. In a recent study, Kaneko et al. ${ }^{8}$, investigated in mice the effect of selective serotonin reuptake inhibitors (SSRI), fluoxetine and paroxetine, on the sensitization induced by methamphetamine, which is a recent model of relapse in psychosis. The authors concluded that treatment with these agents may reverse the methamphetamine- induce behavioral sensitization, and SSRIs would be useful as prophylactic agents in psychosis. Several hypotheses have been reported when focusing on the therapeutic efficacy of antidepressants in DD. Wada and colleagues ${ }^{6}$ suggest a serotonergic dysfunction in DD, specifically in those with a DD somatic type.

However, no double-blind, randomized, controlled trials are available in DD, and psychopharmacological approaches are guided mainly by case reports and schizophrenia in general.

Previous prospective and retrospective studies, case-registers and reports, reviews and meta-analysis included heterogeneous populations with respect to demographic data, delusional type and other clinical features. Due to the paucity of information, no data on antidepressant efficacy in DD can be concluded other than a lower prescription rate than expected.

Further investigations are needed to clarify the role of mood disturbances in the interpersonal, social and occupational functioning in DD and to provide more information for a better therapeutic approach. 


\section{Conclusions}

A high frequency of comorbid depression in DD and a low antidepressants prescription rate has been found. Thus, we conclude that patients with DD should be treated with antidepressants concomitantly when necessary.

Large prospective, controlled and randomized studies in DD are needed to better clarify the efficacy and tolerability profile of antidepressants in the treatment of patients suffering from this chronic delusional disease, as studied in schizophrenic patients of an elderly age.

\section{Limitations}

(1) The possibility of having not found relevant literature regarding this field, specially published and written in languages unknown by the authors, due to the qualitative nature of this review.

(2) The different nature of "depressive" comorbidity, ranging in some studies from minor symptoms attached to an insignificant functional impact to cases suffering from a full grown depressive illness. Only a number of authors describe specifically what kind of depressive symptoms or syndromes qualify in their studies. However, comorbid depressive disorders accountered in the reviewed studies were diagnosed according to DSM-IV-TR criteria.

\section{Conflict of interest}

Dr Alexandre González-Rodríguez has received a research grant from Hospital Clinic of Barcelona (Premi Emili Letang). For the remaining authors there are no conflicts of interest.

\section{References}

1. Kendler KS. Demography of paranoid psychosis (delusional disorder): a review and comparison with schizophrenia and affective illness. Arch Gen Psychiatry 1982; 39: 890902.

2. Kendler KS. The nosologic validity of paranoia (simple delusional disorder). A review. Arch Gen Psychiatry 1980; 37: 699-706.

3. Kendler KS, Tsuang MT. Nosology of paranoid schizophrenia and other paranoid psychoses. Schizophr Bull 1981; 7: 594-610.

4. American Psychiatric Association. Diagnostic and Statistical Manual of Mental Disorders (DSM-III-R). Washington (DC): American Psychiatric Association; 1987.

5. American Psychiatric Association. Diagnostic and Statistical Manual of Mental Disorders (DSM-IV-R). Washington (DC): American Psychiatric Association; 1994.

6. Wada T, Kawakatsu S, Komatani A, Okuyama N, Otani K. Possible association between delusional disorder, somatic type and reduced regional cerebral blood flow. Prog Neuropsychopharmacol Biol Psychiatry 1999; 23: 353-357.

7. Kendler KS, Davis KL. The genetics and biochemistry of paranoid schizophrenia and other paranoid psychoses. Schizophr Bull 1981; 7: 689-709.

8. Kaneko Y, Kashiwa A, Ito T, Ishii S, Umino A, Nishikawa T. Selective serotonin reuptake inhibitors, fluoxetine and paroxetine, attenuate the expression of the established behavioral sensitization induced by methamphetamine. Neuropsychopharmacology 2007; 32: 658-664.

9. Maina G, Albert U, Badà A, Bogetto F. Occurrence and clinical correlates of psychiatric co-morbidity in delusional disorder. Eur Psychiatry 2001; 16: 222-228.

10. de Portugal E, Martinez C, Gonzalez N, Del Amo V, Haro JM, Cervilla JA. Clinical and cognitive correlates of psychiatric comorbidity in delusional disorder outpatients. Aust N Z J Psychiatry 2011; 45: 416-425.

11. Marino C, Nobile M, Bellodi L, Smeraldi E. Delusional disorder and mood disorder: can they coexist? Psychopathology 1993; 26: 53-61.

12. Munro A. The classification of delusional disorders. Psychiatr Clin North Am 1995; 18: 199-212.

13. Kepska A, Hawro T, Krupinska-Kun M, Zalewska A. Somatic-type delusional disorder: a case report and comments. Acta Derm Venereol 2011; 91: 193-194.

14. Manschreck TC, Khan NL. Recent advances in the treatment of delusional disorder. Can J Psychiatry 2006; 51 : 114-119. 
15. Yassa R, Suranyi-Cadotte B. Clinical characteristics of late-onset schizophrenia and delusional disorder. Schizophr Bull 1993; 19: 701-707.

16. Hsiao MC, Liu CY, Yang Y-Y, Yeh EK. Delusional disorder: retrospective analysis of 86 Chinese outpatients. Psychiatry Clin Neurosci 1999; 53: 673-676.

17. Bömmer I, Brüne M. Neuropsychological aspects of delusional disorders. Characteristic attributional style or cognitive deficit? Nervenarzt 2007; 78: 796-801.

18. Grover S, Biswas P, Avasthi A. Delusional disorder: Study from North India. Psychiatry Clin Neurosci 2007; 61: 462-470.

19. de Portugal E, Gonzalez N, Haro JM, Autonell J, Cervilla JA. A descriptive case-register study of delusional disorder. Eur Psychiatry 2008; 23: 125-133.

20. de Portugal E, Gonzalez N, Vilaplana M, Haro JM, Usall J, Cervilla JA. An empirical study of psychosocial and clinical correlates of delusional disorder: the DELIREMP study. Rev Psiquiatr Salud Ment 2009; 2: 72-82.

21. de Portugal E, Gonzalez N, Vilaplana M, Haro JM, Usall J, Cervilla JA. Gender differences in delusional disorder: Evidence from an outpatient sample. Psychiatry Res 2010; 15: 235-239

22. Vicens V, Sarro S, McKenna PJ. Comorbidity of delusional disorder with bipolar disorder: report of four cases. J Affect Disord 2011; 134: 431-433.

23. Wustmann T, Pillmann F, Friedemann J, Piro J, Schmeil A, Marneros A. The clinical and sociodemographic profile of persistent delusional disorder. Psychopathology 2012; 45: 200-202.

24. Wustmann T, Pillmann, Marneros A. Gender-related features of persistent delusional disorders. Eur Arch Psychiatry Clin Neurosci 2011; 261: 29-36

25. Hayashi H, Oshino S, Ishikawa J, Kawakatsu S, Otani K. Paroxetine treatment of delusional disorder, somatic type. Hum Psychopharmacol 2004; 19: 351-352.

26. Hayashi H, Akahane T, Suzuki H, Sasaki T, Kawakatsu $\mathrm{S}$, Otani K. Successful treatment by paroxetine of delusional disorder, somatic type, accompanied by severe secondary depression. Clin Neuropharmacol 2010; 33: 48-49.

27. Nicolato R, Corrêa H, Romano-Silva MA, Teixeira AL Jr. Delusional parasitosis or Ekbom syndrome: a case series. Gen Hosp Psychiatry 2006; 28: 85-87.

28. Dimopoulos NP, Mitsonis CI, Psarra VV. Delusional disorder, somatic type treated with aripiprazol- mirtazapine combination. J Psychopharmacol 2008; 22: 812-814.
29. Quarantini Lde C, Rosário-Campos MC, BatistaNeves SC, Miranda-Scippa A, Sampaio AS. Use of fluoxetine in somatic delusional disorder. Rev Bras Psiquiatr 2004; 26: 138.

30. Alexander J. SSRIs as a treatment alternative for monosymptomatic delusional disorders. Aust N Z J Psychiatry 2010; 44: 295-296.

31. Boggild AK, Nicks BA, Yen L, Van Voorhis W, McMullen R, Buckner FS, et al. Delusional parasitosis: six-year experience with 23 consecutive cases at an academic medical center. Int J Infect Dis 2010; 14: 317-321.

32. Otani K, Miura Y, Suzuki A, Kinoshita O. Effectiveness and safety of milnacipran treatment for a patient with delusional disorder, somatic type taking multiple medications for concomitant physical diseases. Clin Neuropharmacol 2010; 33: 212-213.

33. Sondheimer A. Clomipramine treatment of delusional disorder- somatic type. J Am Acad Child Adolesc Psychiatry 1988; 27: 188-192.

34. Rudden M, Sweeney J, Frances A. Diagnosis and clinical course of erotomanic and other delusional patients. Am J Psychiatry 1990; 147: 625-628.

35. Yamada N, Nakajima S, Noguchi T. Age at onset of delusional disorder is dependent on the delusional theme. Acta Psychiatr Scand 1998; 97: 122-124.

36. Goulia P, Mantas C, Bassukas ID, Hyphantis T. Treatment with risperidone and venlafaxine of a patient with double-coded diagnosis of body dysmorphic disorder and delusional disorder somatic type. Hippokratia 2011; 15: 286-287.

37. Jordan HW, Lockert EW, Johnson-Warren M, Cabell C, Cooke T, Greer W, et al. Erotomania revisited: thirty-four years later. J Natl Med Assoc 2006;98:787-793.

38. Kennedy N, McDonough M, Kelly B, Berrios GE. Erotomania revisited: clinical course and treatment. Compr Psychiatry 2002; 43: 1-6.

39. Montross L, Kasckow J, Golshan S, Solorzano E, Lehman D, Zisook S. Suicidal ideation and suicide attempts among middle-aged and older patients with schizophrenia spectrum disorders and concurrent subsyndromal depression. J Nerv Ment Dis 2008; 196: 884-890.

Corresponding author:

Alexandre González-Rodríguez

170, Villarroel Street

08036 Barcelona (Spain)

Tel. +34619404862

E-mail: alexgonzalezrguez@gmail.com 